\title{
A Location Map Reduced Reversible Data Hiding Method Using Prediction Error Modification
}

\author{
Yanwei $\mathrm{Li}^{1, \mathrm{a}}$, Mei-Chen $\mathrm{Wu}^{2, \mathrm{~b}}$, Tung-Shou Chen ${ }^{3, \mathrm{c}}$ and Wien Hong ${ }^{4, \mathrm{~d}}$ \\ ${ }^{1}$ Department of Information Engineering, Zhengzhou Chenggong University of Finance and \\ Economics, China \\ ${ }^{2,4}$ Department of Information Management, Yu Da University, Taiwan \\ ${ }^{3}$ Department of Computer Science and Information Engineering, National Taichung University of \\ Science and Technology, Taiwan
}

aliyanweicc@gmail.com, bmcwu0715@gmail.com, ttschen@gmail.com, dwienhong@ydu.edu.tw

Keywords: Reversible data hiding, Histogram shifting, Overflow/Underflow

\begin{abstract}
We propose a reversible data hiding technique to improve Hong and Chen's (2010) method. Hong and Chen divide the cover image into pixel group, and use reference pixels to predict other pixel values. Data are then embedded by modifying the prediction errors. However, when solving the overflow and underflow problems, they employ a location map to record the position of saturated pixels, and these pixels will not be used to carry data. In their method, if the image has a plenty of saturated pixels, the payload is decreased significantly because a lot of saturated pixels will not joint the embedment. We improve Hong and Chen's method such that the saturated pixels can be used to carry data. The positions of these saturated pixels are then recorded in a location map, and the location map is embedded together with the secret data. The experimental results illustrate that the proposed method has better payload, will providing a comparable image quality.
\end{abstract}

\section{Introduction}

Data hiding is technique to use a digital media for conveying secret message. Digital media such as texts, audios, videos and images can be used as carriers. The digital image is often used as a cover object because it is available from many digital devices. Moreover, the human eyes are insensitive to the minor change of the pixel value. Data hiding can be categorized into reversible [1] and non-reversible [2]. Reversible data hiding is capable of restoring the original image after extracting the embedded message. On the other hand, the non-reversible data hiding method can not recover the cover image. The image quality is often measured by PSNR. The PSNR and payload are two important factors for a data hiding method. For most data hiding method, an increased in payload will result in a decrease in PSNR. As a result, much research has been done to enhance the payload and image quality. Barton (1997) [3] proposed a data hiding method in his pattern, which is considered as an early model of reversible methods. Tian (2003) [4] and Alattar (2004) [5] proposed reversible data hiding methods based on the expansion of pixel differences for data embedment. Because the expansion of pixel differences may cause a large distortion, their methods cannot offer a high quality stego image. Ni et al. (2006) [6] proposed a new method to enhance the image quality. They embed the secret data by shifting the image histogram. However, the payload of Ni et al.'s method is limited by the peak height of the image histogram.

Thodi and Rodriguez (2007) [7] combined Tian and Ni et al.'s methods and proposed a new reversible method. However, their method is based on the expansion of prediction errors. As a result, a high image quality cannot be achieved. Tsai et al. (2009) [8] divided the image into $3 \times 3$ pixel groups, and used the center pixel of a group to predict other pixel values in the same group. Data are embedded by shifting the prediction errors. Although Tsai et al.'s method offers a high image quality; the payload can be further increased by using a better predictor. In the same year, Hong and Chen [9] also proposed a new method using a bi-linear predictor and had better embedding efficiency over Tsai et al.'s method. They divide image into blocks of $3 \times 3$ pixels, and select the center pixel of each group as the reference pixel. Other pixels rather than the center pixels are 
predicted using the current reference pixel and other four neighboring reference pixels. The layout of the reference pixel and the prediction formula are shown in Fig. 1. Two peaks are obtained from the prediction error histogram. Data are then embedded using the histogram shifting technique. Although Hong and Chen's method provides better embedding performance over Tsai et al.'s method, the mechanism they used to process the overflow and underflow pixels may decrease the pure payload. In their method, a location map (LM) is used to record the saturated pixels (pixel value is 255 or 0 ), and the recorded saturated pixels will not be used to carry data. As a result, Hong and Chen's method may loss significant payload if the cover image has a considerable number of saturated pixels.

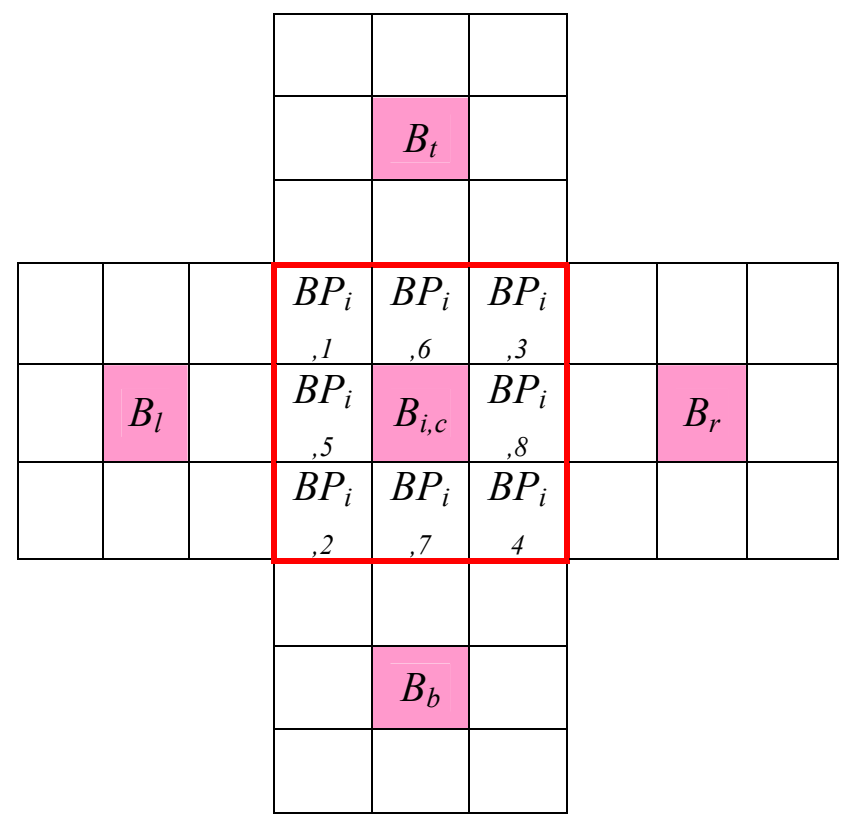

$$
\begin{aligned}
& B P_{i, 1}=\operatorname{round}\left(\left(B_{l}+B_{t}+B_{i, c}\right) / 3\right) \\
& B P_{i, 2}=\operatorname{round}\left(\left(2 \times B_{i, c}+B_{l}\right) / 3\right) \\
& B P_{i, 3}=\operatorname{round}\left(\left(B_{l}+B_{i, c}+B_{b}\right) / 3\right) \\
& B P_{i, 4}=\operatorname{round}\left(\left(2 \times B_{i, c}+B_{t}\right) / 3\right) \\
& B P_{i, 5}=\operatorname{round}\left(\left(2 \times B_{i, c}+B_{b}\right) / 3\right) \\
& B P_{i, 6}=\operatorname{round}\left(\left(B_{t}+B_{i, c}+B_{r}\right) / 3\right) \\
& B P_{i, 7}=\operatorname{round}\left(\left(2 \times B_{i, c}+B_{r}\right) / 3\right) \\
& B P_{i, 8}=\operatorname{round}\left(\left(B_{i, c}+B_{b}+B_{r}\right) / 3\right)
\end{aligned}
$$

Fig. 1. Schematic diagram of reference pixels and to-be-predicted pixels.

\section{Propose method}

In this section, we improve Hong and Chen's method by proposing a new mechanism to process the overflow and underflow problems more efficiently. In the proposed method, the saturated pixels valued 255 and 0 are subtracted and added by one, respectively. A location map LM is then used to record the positions of these recorded pixels. The $L M$ is then compressed [11] and is embedded together with the secret data [12]. Because the cover image is pre-processed, every pixel in the cover image can be used for data embedment. Therefore, the proposed method will provide a payload than Hong and Chen's method. Moreover, we also proposed a new method to better predicted pixel values. As a result, the payload can be further increased. The detailed embedding and extraction procedures are listed as follows.

\section{Embedding Procedure}

Input: Cover image $I$, secret message $S$

Output: Stego image $I^{\prime}$, peaks $P_{L}, P_{R}$, message length $|S|$, size of $L M|L M|$

Step 1. The pixels in the cover image are pre-processed, i.e., pixels valued 255 are subtracted by 1 and pixels valued 0 are added by 1 . The positions of these pre-processed pixels are recorded in a location map, and the location map is compressed to obtain $L M$. 
Step 2. Divide $I$ into blocks of $3 \times 3$ pixels. We denote the $\mathrm{i}$-th block as $B_{i}$. The reference pixel of $B_{i}$ is denoted by $B_{i, c}$. Pixels other than $B_{i, c}$ are denoted by $\left\{B_{i, j}\right\}_{j=1}^{8} \cdot\left\{B_{i, j}\right\}_{j=1}^{8}$ are predicted using the formula shown in Fig. 2. Let the predicted results be pixels $\left\{B P_{i, j}\right\}_{j=1}^{8}$.

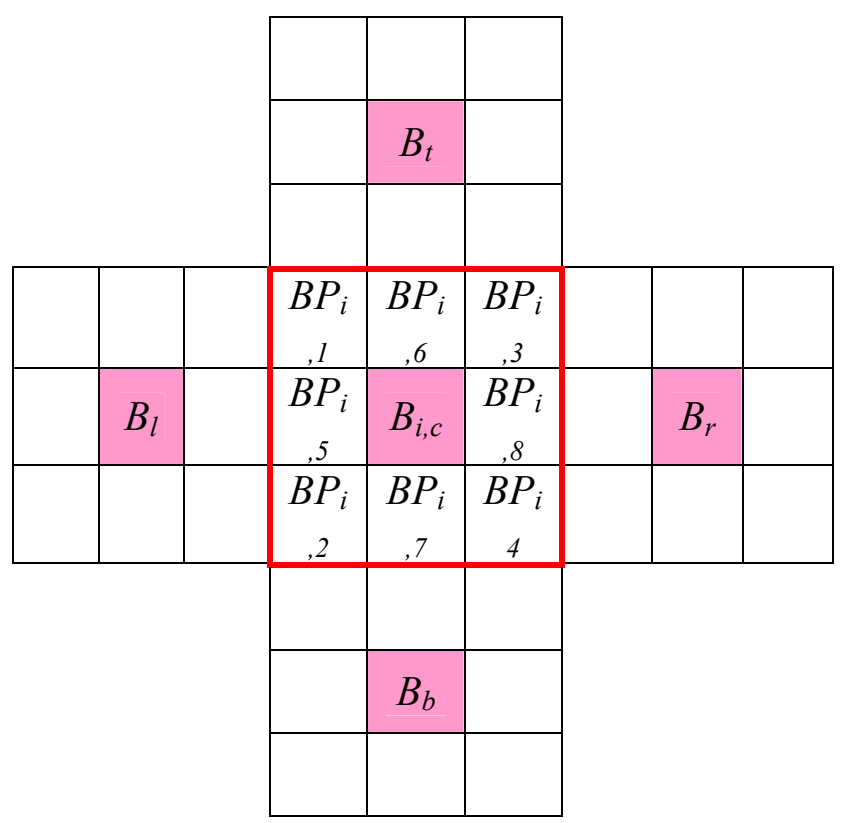

$$
\begin{aligned}
& B P_{i, 1}=\operatorname{round}\left(\left(B_{l}+B_{t}+B_{i, c}\right) / 3\right) \\
& B P_{i, 2}=\operatorname{round}\left(\left(B_{l}+B_{i, c}+B_{b}\right) / 3\right) \\
& B P_{i, 3}=\operatorname{round}\left(\left(B_{t}+B_{i, c}+B_{r}\right) / 3\right) \\
& B P_{i, 4}=\operatorname{round}\left(\left(B_{i, c}+B_{b}+B_{r}\right) / 3\right) \\
& B P_{i, 5}=\operatorname{round}\left(\left(B P_{i, 1}+B P_{i, 2}+B_{i, c}\right) / 3\right) \\
& B P_{i, 6}=\operatorname{round}\left(\left(B P_{i, 1}+B_{i, c}+B P_{i, 3}\right) / 3\right) \\
& B P_{i, 7}=\operatorname{round}\left(\left(B P_{i, 2}+B_{i, c}+B P_{i, 4}\right) / 3\right) \\
& B P_{i, 8}=\operatorname{round}\left(\left(B P_{i, 3}+B_{i, c}+B P_{i, 4}\right) / 3\right)
\end{aligned}
$$

Fig. 2. Schematic diagram of reference pixels and to-be-predicted pixels.

Step 3. Next, we calculate the prediction errors $\left\{E_{i, j}\right\}_{j=1}^{8}=\left\{B_{i, j}\right\}_{j=1}^{8}-\left\{B P_{i, j}\right\}_{j=1}^{8}$ for every block to obtain the prediction error histogram.

Step 4. Two peaks, $P_{L}$ and $P_{R}$ are obtained from the constructed histogram. We assume $P_{L}<P_{R}$. Scan the prediction errors. If $E_{i, j}=P_{L}$ or $E_{i, j}=P_{R}$, extract a bit $s$ from $S$ and use Eq. 1. to embed the extracted bit

$$
E_{i, j}^{\prime}= \begin{cases}E_{i, j} & \text { if } s=0, \\ E_{i, j}-1 & \text { if } s=1 \text { and } E_{i, j}=P_{L}, \\ E_{i, j}+1 & \text { if } s=1 \text { and } E_{i, j}=P_{R} .\end{cases}
$$

Otherwise, the scanned prediction error has to be shifted by using Eq. 2:

$$
E_{i, j}^{\prime}= \begin{cases}E_{i, j}-1 & \text { if } E_{i, j}<P_{L}-1, \\ E_{i, j}+1 & \text { if } E_{i, j}>P_{R}+1,\end{cases}
$$

Step 5. The stego pixels can be obtained by calculating $\left\{B_{i, j}^{\prime}\right\}_{j=1}^{8}=\left\{E_{i, j}^{\prime}\right\}_{j=1}^{8}+\left\{B P_{i, j}\right\}_{j=1}^{8}$.

Step 6. Repeat Steps 3-5 until all the secret data are embedded and the stego image $I^{\prime}$ is obtained.

\section{Extraction Procedure}

Input: Stego image $I^{\prime}$, peak $P_{L}, P_{R}$, message length $|S|$, size of $L M|L M|$

Output: Recover image $I$, extract message $S$ 
Step 1. Divide $I^{\prime}$ into blocks of $3 \times 3$ pixels and obtain the predicted value $\left\{B P_{i, j}^{\prime}\right\}_{j=1}^{8}$. This procedure is the same as Step 2 in the embedding procedure. The prediction errors are calculated by $\left\{E_{i, j}^{\prime}\right\}_{j=1}^{8}=\left\{B_{i, j}^{\prime}\right\}_{j=1}^{8}-\left\{B P_{i, j}^{\prime}\right\}_{j=1}^{8}$.

Step 2. Visit the prediction errors $\left\{E_{i, j}^{\prime}\right\}_{j=1}^{8}$. If $E_{i, j}^{\prime}=P_{R}, P_{L}, P_{R}+1$, or $P_{L}-1$, a message bit $s$ can be extracted using Eq. 3:

$$
s= \begin{cases}0 & \text { if } E_{i, j}^{\prime}=P_{L} \text { or } E_{i, j}^{\prime}=P_{R}, \\ 1 & \text { if } E_{i, j}^{\prime}=P_{L}-1 \text { or } E_{i, j}^{\prime}=P_{R}+1 .\end{cases}
$$

Otherwise, we have to restore the prediction errors using the following equation:

$$
E_{i, j}= \begin{cases}E_{i, j}+1 & \text { if } E_{i, j}^{\prime}<P_{L}-1, \\ E_{i, j}-1 & \text { if } E_{i, j}^{\prime}>P_{R}+1 .\end{cases}
$$

Step 3. The cover pixels can be obtained by calculating $\left\{B_{i, j}\right\}_{j=1}^{8}=\left\{E_{i, j}\right\}_{j=1}^{8}+\left\{B P_{i, j}\right\}_{j=1}^{8}$.

Step 4. Repeat the extraction and recovery procedures until all the secret data are extracted and the cover image $I$ is restored.

\section{Experimental Results}

In the section, we use eight grayscale test images of size $512 \times 512$ obtained from USC-SIPI [13] and RSP [14] image databases. Among these test images, images a and b possess few saturated pixels, and images c-h possess considerable number of saturated pixels. We use PSNR and Payload to compare the performance of the proposed method and other Hong and Chen's method.

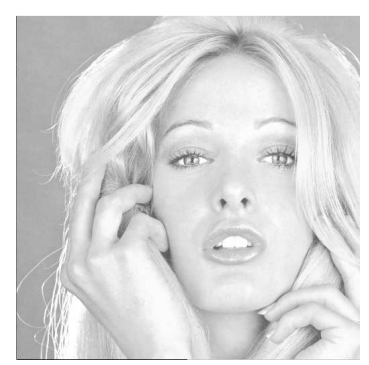

a

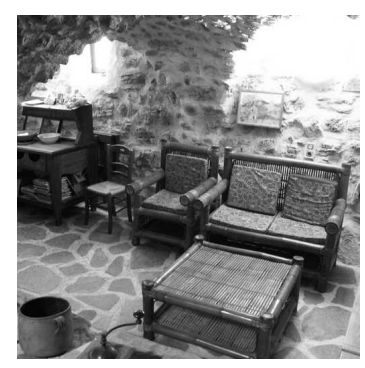

e

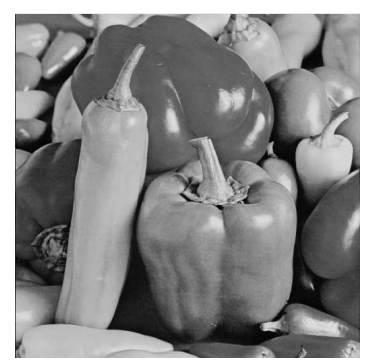

$\mathrm{b}$

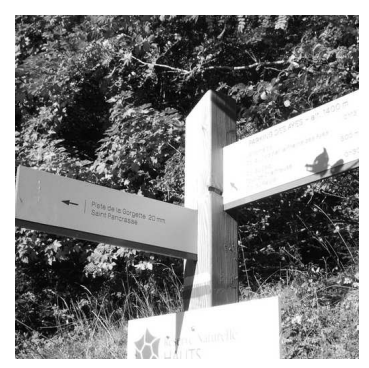

$\mathrm{f}$

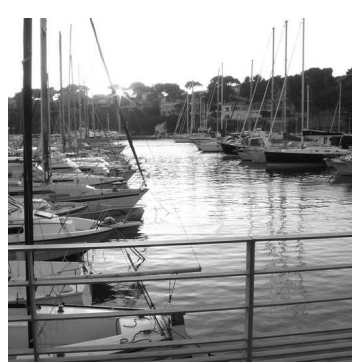

C

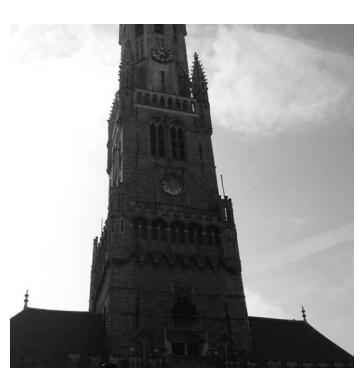

g

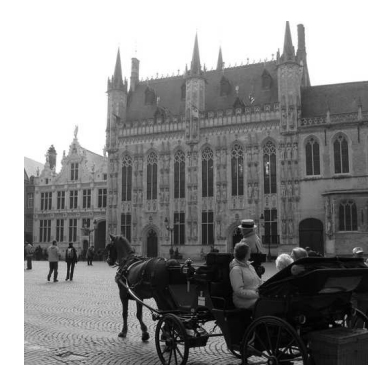

d

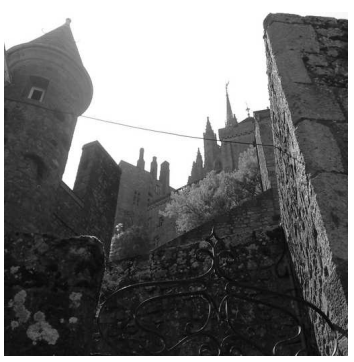

$\mathrm{h}$

Fig 3. The eight grayscale test images.

As shown in Table 1, when the test images possess fewer saturated pixels (images a and $b$ ), the proposed method provides a higher payload with an insignificant decrease in PSNR. For the test images with considerable amount of saturated pixels (images c-h), the payload of the proposed method is significantly higher than those of Hong and Chen's method with a decrease in image quality around $1 \mathrm{~dB}$. However, the image quality of the proposed method is higher than $48 \mathrm{~dB}$. The human eyes are indistinguishable a change of $1 \mathrm{~dB}$ of PSNR at this level. As a result, the proposed method offers a comparable image quality to Hong and Chen's method while providing a significantly higher payload. 
Table 1. Compare proposed and Hong and Chen's method.

\begin{tabular}{|c|c|c|c|c|}
\hline \multirow{2}{*}{} & \multicolumn{2}{|c|}{ Proposed method } & \multicolumn{2}{c|}{ Hong and Chen's method } \\
\cline { 2 - 5 } & $P S N R$ & Payload & PSNR & Payload \\
\hline $\mathrm{a}$ & 49.27 & 48267 & 49.33 & 48100 \\
\hline $\mathrm{b}$ & 49.15 & 36436 & 49.15 & 36287 \\
\hline $\mathrm{c}$ & 48.16 & 37350 & 49.74 & 16568 \\
\hline $\mathrm{d}$ & 49.30 & 50674 & 49.96 & 16421 \\
\hline $\mathrm{e}$ & 48.67 & 31483 & 49.31 & 21801 \\
\hline $\mathrm{f}$ & 48.47 & 18911 & 49.42 & 4226 \\
\hline $\mathrm{g}$ & 48.68 & 104858 & 50.46 & 77171 \\
\hline $\mathrm{h}$ & 49.45 & 62057 & 50.20 & 18601 \\
\hline Average & 48.89 & 48755 & 49.70 & 29897 \\
\hline
\end{tabular}

\section{Conclusions}

Hong and Chen's method obtains a good image quality. however, when solving the overflow and underflow problems, their method may result in a decrease in payload because the saturated pixels will not be used for data embedment. We improve Hong and Chen's method such that the saturated pixels can be used to carry data. The experimental results demonstrate that the proposed method has better payload, while providing a comparable image quality.

\section{References}

[1] W. Hong and T.S. Chen, "Reversible Data Embedding for High Quality Images Using Interpolation and Reference Pixel Distribution Mechanism," Journal of Visual Communication and Image Representation, vol. 22, no. 2, pp. 131-140, 2011.

[2] W. Hong, and T.S Chen, "A Novel Data Embedding Method Using Adaptive Pixel Pair Matching," IEEE Transactions on Information Forensics and Security, vol.99, no.1, pp.176-184, 2012.

[3] J.M. Barton, "Method and Apparatus for Embedding Authentication Information within Digital Data," U.S. Patent 5646 997, Jul. 8, 1997.

[4] J. Tian, "Reversible Data Embedding Using a Difference Expansion," IEEE Transactions on Circuits and Systems for Video Technique, vol. 13, no. 8, pp. 890-896, 2003.

[5] A.M. Alattar, "Reversible Watermark Using the Difference Expansion of A Generalized Integer Transform," IEEE Transactions on Image Processing, vol. 13, no. 8, pp. 1147-1156, 2004.

[6] Z. Ni, Y.Q. Shi, N. Ansari, and W. Su, "Reversible Data Hiding," IEEE Transactions on Circuits and Systems for Video Technique, vol. 16, no. 3, pp. 354-362, 2006.

[7] D.M. Thodi and J.J. Rodriguez, "Expansion Embedding Techniques for Reversible Watermarking," IEEE Transactions on Image Processing, vol. 16. no. 3, pp. 721-730, 2007.

[8] P.Y. Tsai, Y.C. Hu, and H.L. Yeh, "Reversible Image Hiding Scheme Using Predictive Coding and Histogram Shifting,” Signal Processing, vol. 89, no. 6, pp. 1129-1143, 2009.

[9] W. Hong, T.S. Chen, and C.W. Shiu, "Reversible Data Hiding for High Quality Images Using Modification of Prediction Errors," Journal of Systems and Software, vol. 82, no. 11, pp. 1833-1842, 2009. 
[10]W. Hong and T.S. Chen, "A Local Variance-Controlled Reversible Data Hiding Method Using Prediction and Histogram-Shifting," The Journal of Systems and Software, vol.83, no. 12, pp. 2653-2663, 2010.

[11]X. Wu and N. Memon, "CALIC-a context based adaptive lossless image codec," IEEE Transactions on Image Processing, vol.4, pp.1890-1893, 1996.

[12]Y.-W. Li and W. Hong, "An Improved Reversible Data Hiding Method Using Alternative Location Map Embedment Strategy," Information Technology Journal, vol.11, no.8,pp. 1109-1114,2012.

[13]USC-SIPI image database. Available: http://sipi.usc.edu/database.

[14] RSP image database, http://dud.inf.tu-dresden.de/westfeld/rsp/rsp.html. 